\title{
LA REFLEXIÓN INTERCULTURAL COMO BASE PARA IMAGINAR UMA EDUCACIÓN DEMOCRÁTICA PARA LOS PUEBLOS AUTÓCTONOS Y PARA LA SOCIEDAD MULTICULTURAL EM AMÉRICA LATINA
}

Prof. José Marín

\section{Resumo}

Nosso texto procura fazer uma reflexão sobre o tipo de educação escolar mais adequado para os povos autóctones e para as sociedades multiculturais da América Latina a partir de uma perspectiva intercultural. Compreendemos a educação intercultural como uma proposta que se realiza a partir de um contexto sociopolítico e cultural que procura orientar os atores educacionais no sentido de reagir contra a subordinação imposta pela cultura dominante e pelos seus sistemas de conhecimento e de valores. Acreditamos que o papel de socialização atribuído à escola pode se constituir em um estímulo eficaz para a necessária descentralização cultural e para contribuir para a construção de uma sociedade democrática na qual impere 0 respeito pela dignidade humana

Pavara-chave: Educação - Intercultural - Saber - Cultura - Professores.

\section{Resumen}

Nuestro texto hace una reflexión sobre la educación escolar que sería adecuada a los pueblos autóctonos y para las sociedades multiculturales de Latinoamérica a partir de una perspectiva intercultural. Concebimos la educación intercultural como una proposición a partir de un contexto sociopolítico y cultural que busca orientar a los actores educativos a reaccionar a la subordinación impuesta por la cultura dominante y sus sistemas de conocimiento y de valores. Acreditamos que el papel de la socialización en la escuela puede constituir una estimulación muy eficaz en la necesaria descentralización cultural y contribuir para la construcción de una sociedad democrática donde impera una respetuosa dignidad humana.

Palabras Llaves: Educación - Intercultural -Saberes - Cultura - Maestro

* José Marín, sociologo y antropólogo peruano, es Doctor en Antropología por la Universidad de La Sorbonne y ha realizado estudios de Post-grado en el Instituto de Altos Estudios de América Latina de Paris. Igualmente es diplomado del Instituto Universitario de Estudios del Desarrollo y de la Academia Internacional del Medio Ambiente de Ginebra. 


\section{Introduocion}

La pregunta central que tratamos de responder y que anima nuestra reflexión, es de saber como podemos imaginar una educación escolar apropiada para los pueblos autóctonos y para las sociedades multiculturales de América Latina a partir de la perspectiva intercultural.

Al final del siglo XX, los pueblos autóctonos exigen el derecho a la autonomía y a la auto-gestión de sus territorios ancestrales y la revalorización de sus lenguas y culturas. Este hecho constituye un movimiento, de una profunda significacion en virtud del cual son reafirmados sus conocimientos, sus saberes y sus modos de aprendizaje. Este hecho tiene una gran importancia en muchas regiones del mundo actual. A principios del mes de noviembre de 1999, la Organización Mundial de la Propiedad Intelectual (OMPI) organizo en Ginebra, una Mesa Redonda sobre: «os saberes tradicionales y la propiedad intelectual indígena», con la asistencia de delegaciones indígenas, representantes estatales, ONGS y con la participacion de delegados de empresas multinacionales.

Actualmente, en la gran mayoría de los paises donde sobreviven estos pueblos, los programas escolares desarrollados por la educación oficial monolingüe y mono-cultural se encuentran confrontadas al desafío de saber, cómo administrar la diversidad lingüística y cultural.

Entre 1989 y el 2002 ha ensenado y colaboradoen la Facultad de Psicología y Ciencias de la Educación de la Universidad y en la Academia Internacional del Medio Ambiente de Ginebra.

Colabora con la UNESCO en el Africa. Ha contribuído igualmente, con la revista italiana «atinoaménica» Análisis Textos y Debate, con la revista «Aninzana» de Caracas, Venezuela y con las Ediciones «Abya - Yala»de Quito, Ecuador.

A partir de esta constatación, nosotros creemos que la educación intercultural puede constituir una de las reflexiónes fundamentales en la elaboración de los programas, que puedan poner en valor las lenguas y las culturas autóctonas, asociándolas a las perspectivas de la cultura occidental. En America Latina el respeto de las lenguas y culturas ya es una consideración que es tomada en cuenta en la mayor parte de programas.

La educación a partir de la reflexión intercultural y bilingüe constituye el vehículo a través del cual se expresan la canalización de la defensa de estos derechos en Africa, Asia en Oceanía, Europa, en América del norte y algunos países de América latina. (LIPKA, J. \& STAIRS, A.; ROSTOWSKI, J., 1973; TEASDALE, 19.

Se denominan pueblos autóctonos o pueblos indígenas, a los primeros ocupantes o a los pueblos originarios de un territorio determinado (TEASDALE, 1994). En 1993, las Naciones Unidas han proclamado "El Decenio de los pueblos autóctonos". 
En Europa, los programas oficiales de educación están confrontados a la existencia de minorías nacionales y étnicas, igualmente, a una población inmigrante y a poblaciones itinerantes, como es el caso de los gitanos, principalmente en Europa del Este. (DERENS,1999).

Actualmente la educación intercultural en Europa está destinada a la población extranjera, pero es necesario abandonar el lugar marginal que esta ocupa y asumir la diversidad sociocultural y lingüística, como finalidad de la educación, más allá de la visión mono-cultural que impregna la educación europea actual. Jurgen GASCHÉ (1999), afirma que : ¿a educación intercultural debe interesar a todos los países que cuentan con poblaciones minoritarias con características sociales, religiosas, lingüísticas y culturales que los diferencian de una sociedad, de una religión, lengua y cultura que pretenden ser representativas de la «nación», en tanto mito unificador proclamado por el Estado».

No se pude hablar de perspectiva intercultural en la educación, sín hacer referencia a la dimensión politica que está a la base de los programas educativos oficiales, impuestos por los Estados-Nación, en relación con la diversidad lingüística y cultural, ignorada por la escuela, asociada a la política de asimilación de las minorias étnicas de América Latina. Nuestro cuadro teórico de referencia esta constituido en principio, por las investigaciones realizadas y publicadas en América Latina en este dominio, principalmente en el Peru, donde he podido visitar el Programa de Formación de Maestros Bilingües (PFMB) iniciado por la Asociación Indígena de Desarrollo de la Selva Peruana (AIDESEP) y el Instituto Pedagógico Superior Loreto (ISPL), actualmente ubicado en Zungarocha, pueblo cercano a la ciudad de Iquitos, en la Amazonía peruana.

Este artículo aborda la problemática de las experiencias en educación bilingüe e intercultural, de una manera introductiva y limitada, por tanto adolece de una serie de limitaciones, y no pretende abordar todas las experiencias existentes, que constituyen un tema amplio y complejo.

La primera parte de nuestro artículo esta consagrado a una breve introducción teórica general, sobre las experiencias interculturales en la educación en el contexto histórico de América del Norte y América Latina. La segunda parte trata brevemente sobre las experiencias de América del Sur, tomando como referencia principal la experiencia peruana. Dimensión histórica del contexto intercultural. Nosotros somos el producto histórico de diferentes mestizajes acaecidos en el curso de la historia de las civilizaciones (LAPLATINE \& NOUS, 1997). Este proceso se desarrolló y se desarrolla en el marco físico de una enorme diversidad ecologica, de la que surgió la diversidad cultural: El hombre pertenece a una sola especie biológica, más allá de todas las jerarquizaciones ideológicas desarrolladas por el racismo, desde la época colonial hasta nuestros días (LANGANEY; VAN BLJENBURG \& SÁNCHEZMAZAS, 1995; MARÍN 1994, 1995).Desde esta perspectiva, toda pretensión de 
pureza biológica y cultural es absurda, cada uno de nosotros posee una historia genética única, partiendo del hecho que todos pertenecemos a la misma especie. La unidad del género humano, es hoy en día, indiscutible.

La historia de las sociedades humanas está asociada a la historia de las migraciones; a los contactos culturales, fuente de múltiples influencias. Estos reencuentros han tenido caracteres diversos: ya sea de rechazo y confrontaciones o de intercambios pacificos y aprendizajes mutuos. Las culturas se han construido y modificado en contextos dinámicos por las colectividades y los individuos; en tanto que participantes de interacciones de su propia historia, sometidos a influencias y a presiones múltiples, que han condicionado la construcción de sus identidades.

Actualmente, con el proceso de globalización de la economía, reforzada por la revolución tecnológica de las comunicaciones, que permiten la circulación de la información, se multiplican los contactos entre los pueblos en diversos dominios; aún cuando la ideología neo-liberal proclame el libre tránsito de las mercancías, los gobiernos de los países ricos imponen una política de inmigración restrictiva y discriminatoria, al limitar el libre tránsito de las personas. El planeta que habitamos se convierte en una «aldea global» con insalvables contradicciones y distancias socioeconomicas que producen repercusiones perversas, tanto en el sur, como en el norte (OMAN, 1996; HALLAK, 1998; FORRESTER, 1996; UGARTECHE, 1999). Más allá de los intereses de la geopolítica, todos nosotros, los más de seis mil millones de seres humanos, sobrevivimos sobre el mismo cielo y habitamos en la misma «casa». La pregunta central a responder es: cómo hacer para vivir juntos? Cómo hacer para respetarnos y aprovechar de nuestra diversidad cultural mutuamente? Este es el desafío universal que nos propone la dimensión intercultural, desafío ineludible, que tiene que afrontar la sociedad humana contemporánea, (DEMORGON, 2000; DEMORGON, 1999; DEMORGON \& LIPIANSKI, HUNTINGTON, 1997; LEMPEN, 1999; WARNIER, 1999). La manera como se desarrollan las sociedades contemporáneas, está impregnada por la interculturalidad, en contextos muy diferentes: que van desde las descolonizaciones, las guerras, los genocidios, las crísis, las recesiones económicas, la construcción de bloques hegemónicos, las migraciones hasta la actual globalización, que provoca mutaciones y contradicciones socioeconómicas y culturales, y la destrucción del mercado de trabajo y las exclusiones. Estos contextos asocian lo trágico a lo complejo, donde se articulan lo local con lo mundial y que provocan la pérdida de los puntos de referencia sobre los cuales sobrevivían gran parte de nuestras sociedades (HESSE, 1998).

La perspectiva Intercultural entre identidad y alteridad. La reflexión intercultural implica una preocupacion fundamental, que es la de imaginar una pedagogía apropiada a las sociedades multiculturales. La comunicación se construye entonces sobre la base del respeto de la diversidad cultural y nos 
permite desarrollar una percepción del mundo, como un lugar histórico a compartir entre todos los seres vivientes. El hecho de reconocer y de respetar la existencia de la alteridad -de los otros- nos obliga a reflexionar en las interrogantes, sobre la calidad de nuestras relaciones con los demás. Esta reflexión implica a la sociedad en que vivimos, a sus contradicciones y fundamentalmente a la escuela, en tanto institución intermediaria entre el estado y la sociedad, y en consecuencia intermediaria, de la diversidad cultural y lingüística existente en la misma.

Cada cultura, construye su propia visión del mundo y en consecuencia su propia racionalidad, pretendiendo considerarla como universal, dentro de la perspectiva etnocéntrica que impregna a cada cultura. Como afirmaba Carmel CAMLLERI (1993, p.35): ¿as culturas se centran sobre si mísmas, todas proceden de un modelo de partida con relación al cual, sus representaciones y sus valores son justificados)(traducción del autor).

La implicación social de nuestra vida cotidiana y la interacción de unos, con los otros, ponen en discusion nuestras identidades. Esta confrontación con nosotros mismos engendra una inseguridad que impide la descentración cultural indispensable a una dinámica intercultural, teniendo en cuenta la dificultad que encuentra la reflexión intercultural por la complejidad de la realidad y las exigencias éticas que acompanian esta práctica.

No podemos limitarnos a la lucha contra los prejuicios, la intolerancia, el etnocentrismo, el nacionalismo y el racismo. No podríamos contentarnos tampoco, con la descripción y comparación, sín comprometernos en la búsqueda y la construcción de mecanismos que favorescan el respeto de la pluralidad de nuestras sociedades. El sentimiento de un mutuo reconocimiento, es sin duda el punto de partida.

La perspectiva de la reflexión intercultural es aplicable en diferentes dominios, partiendo del reencuentro entre la identidad y la alteridad, del diálogo entre nosotros con los otros, dentro de una perspectiva que nos permita reconocer los rasgos comunes que impregnan a todos los seres humanos.

Esta reflexión nos permite igualmente, imaginar la existencia de otras formas de percepción de los conocimientos y de la interpretación de la realidad. El enfoque interdisciplinario utilizado por la reflexion intercultural, nos permite el reencuentro con los múltiples rostros de la sociedad y de su complementariedad, en vista de la integración democrática y participativa que buscamos.

Actualmente, asistimos al fin de ciertas concepciones vehículadas por el etnocentrismo, desde la época colonial, presentadas como "verdades científicas « como aquella de la existencia de las razas y la jerarquización biológica y cultural que de estas afirmaciones se desprenden. No se puede afirmar ni biológica, ni genéticamente, la existencia de razas. No se puede constituir una «aza», ni siquiera al interior de una familia, por que cada persona, es una 
historia biológica y genética única. (LANGANEY; VAN BLJENBURGH; SÁNCHEZMAZAS, 1995).

Todos los seres humanos pertenecen a la misma especie y todos somos diferentes. Esta diversidad no es un defecto, sino un rasgo determinante que preserva la especie humana en su conjunto. La especificidad de la naturaleza humana es precisamente el hecho de inscribirse en culturas con rasgos particulares, que no tienen ninguna relación con un patrimonio genético. Las diferencias socioeconómicas entre los individuos y los pueblos no se justifica ni biológicamente, ni culturalmente(MARIN, 1994).

Actualmente, la globalización de la economia capitalista controla tan bien el dominio de la información y las comunicaciones que sustentan la imposición de una masificación y «standarización» cultural del modelo de la sociedad norteamericana. Proceso que tan bien se traduce en el debilitamiento económico y político de los Estados-nacionales, que provoca paralelamente, la emergencia de identidades étnicas, como expresión de una resistencia a esta hegemonía. Proceso que favorece, entre otros, la creación de programas educativos alternativos en América Latina (GASCHÉ, 1998b).

En Europa, se manifiesta un doble movimiento de reinvindicaciones. De una lado, ciertas regiones se consideran oprimidas por un Estado centralizador y reínvindican el derecho a sus lenguas y a la revalonización de sus culturas, en las instituciones educativas y culturales(es el caso, entre otros, de los Catalanes en España y de las minorías nacionales húngaras en Rumania). De otro lado, ciertas regiones recurren algunas veces a estrategias de resistencia militar, en las que la exigencia del respeto a sus lenguas y culturas está asociada a su demanda de independencia política (como es el caso de los Vascos en España y los Corsos en Francia (FERRER, 1998; GASCHÉ, 1998b; PÉREZ, 1998; SALVI, 1973).

Un segundo movimiento en el ámbito europeo es el de las poblaciones de inmigrantes, que buscan proteger y perpetuar sus herencias lingüísticas, culturales y religiosas en los paises de su nueva residencia.

En la actualidad, asistimos a otros escenarios de conflictos llamados « inter-étnicos « que han surgido despues del desmembramiento de la ex Unión Soviética, dando lugar a conflictos armados, como es el caso de la lucha por la independencia de Tchechenia y Abkasia en el Cáucaso y en otros países del Asia central.

El ejemplo más dramático de las tensiones provocadas por la intolerancia de la diversidad religiosa, lingüística y cultural, de la parte de un nacionalismo extremo se manifiesta en el drama de la purificación étnica, que se desencadenó con el desmembramiento de la Confederacion Yugoslava Serbia, Croacia y Bosnia) a principios de los años 90 y últimamente en el drama vivido entre serbios y kosovares, y a las agresiones sufridas por las minorías gitanas en manos de serbios y kosovares (DERENS, 1999).

Es importante igualmente, senalar el caso dramático del pueblo Kurdo 
que lucha contra el Estado nacionalista turco, que nos demuestra la incoherencia del discurso de quienes pretenden defender los Derechos Humanos en Yugoslavia, dejando realizar en total impunidad al Estado turco, aliado de las grandes potencias occidentales, su propia purificación étnica. Lo que nos comprueba que los intereses geopolíticos y económicos son prioritarios, ante cualquier discurso humanitario»(PICARD, 1991).

La situación mundial contemporánea está caracterizada por las múltiples mutaciones que provocan los grandes cambios económicos, sociales y culturales.

En esta perspectiva, la reflexión intercultural recupera su dimensión histórica, más allá de la problemática educativa, psicológica y migratoria, esta reflexión nos ayuda a comprender más ampliamente el respeto de la diversidad, de la pluralidad y de la difícil gestión de la democracia. En estas condiciones, la reflexión intercultural nos permite comprender mejor este proceso y puede facilitar la posibilidad de imaginarnos, cómo vivir juntos.

La ruptura y el divorcio entre el Estado y la sociedad, que se supone que el Estado representa, se manifiestan en el ámbito planetario. En Aménica Latina, se caracteriza por la emergencia de las identidades indígenas que buscan defender sus derechos, el caso del Movimiento Zapatista de Chiapas en México, donde el modelo político de Estado-Nación, debilitado por los efectos de la globalización, es puesto en discusión, en tanto, modelo político de Estado, incapaz de representar la sociedad multicultural mexicana. (CAMPA MENDOZA, 1999; DÍAZ POLANCO, 1997).

Las revueltas indígenas en el Ecuador, reclaman el derecho a la revalorización de sus lenguas y culturas a través de programas educativos apropiados.

El caso de Chile, es un buen ejemplo de esta tendencia actual, donde el regreso a las tradiciones democráticas, propician las condiciones para la Educación Intercultural Bilingüe a partir de la promulgación de la Ley Orgánica Constitucion de Ensenanza (LOCE) promulgada en marzo de 1990 y la otra ley 19253 del ano 1993, llamada también Ley Indígena. Esta Ley tiene la virtud, de reconocer por primera vez la multi-etnicidad de Chile y expresa el reconocimiento y valoración de las etnias Mapuches, Aymaras, Rapa Nui, Atacameros, Colla, Kawashar y Yagana, a las cuales, el Estado se compromete jurídicamente a respetar, proteger y promover programas para facilitar el desarrollo de los indígenas. Igualmente esta Ley, se propone proteger los territorios indígenas preservando el equilibrio ecológico. El Título IV acerca de la Cultura y la Educación Indígena, es el que contempla la necesidad de reconocer y proteger las culturas e idiomas indigenas (MRANDA VEGA, 1996).

Un buen ejemplo de este proceso lo constituye, el Programa de Formación de Maestros Mapuches de la Universidad Catolica de Temuco, que ofrece en su programa un diploma en educación con mención en Educación intercultural y Bilingüe. Este programa esta compuesto de tres módulos, y uno 
de ellos esta destinado a la Educación Mapuche, lengua y cultura; historia y sociedad; articulación Institucional y social, y finalmente Metodología Intercultural. Este módulo está basado en una orientación intercultural, cuyo objetivo es «ormar maestros de orígen mapuche, capaces de actuar en contextos pluri-etnicos y pluri-culturales, teniendo como finalidad orientar la acción educativa, en función de la valorización de los pueblos mapuches y su cultura. El programa tiene como objetivo igualmente, desarrollar en sus estudiantes, la capacidad de afirmar sus identidades para participar en el mundo contemporáneo (COCCHI, 2000; QUILLAQUEO, 1999).

En Venezuela, donde la Constitución de 1961 declaraba a los indígenas en vías de extinción, el actual gobierno de Hugo Chávez, elegido recientemente, participo en marzo de 1998, en una reunión de la Confederación Indígena de Venezuela (CONIVE), organización representativa de treinta pueblos indígenas, donde suscribió un documento titulado «n compromiso para la Historia» en el cual se compromete a saldar la deuda histórica de Venezuela con los pueblos indígenas del país.

Actualmente, existe una representación indígena en el Parlamento venezolano y el respeto de las lenguas y las culturas indígenas al interior de la política educativa gubernamental que están planteadas en el proyecto de la Constitución venezolana, en el capítulo VIII referente a los derechos de los pueblos indígenas, artículo 119: «El Estado reconocerá la existencia de los pueblos y comunidades indígenas, su organización social, política y económica, sus culturas, usos y costumbres, idiomas y religiones, así como su habitat y derechos originarios sobre las tierras que ancestral y tradicionalmente ocupan y que son necesarias para desarrollar y garantizar sus formas de vida». Solo la realidad histórica nos confirmara si todos estos nobles principios jurídicos serán aplicados en la práctica.

\section{ElConcepto DeCultura Y La DescentraciónCulturalComo Punto De Partida DelIntercultural.}

El concepto de cultura nos permite una mejor comprensión de la naturaleza humana. El hombre, es el producto de esta herencia natural del medio cultural, en el cual ha sido socializado. El hombre surge de un vasto proceso de acumulación ilimitado de conocimientos, según las diferencias de posibilidades y de experiencias que constituyen la historia de cada uno, alejado de todo determinismo. Cada uno de nosotros, es el producto de un contexto cultural, en el cuales capaz de reconocerse y sobre el cual construye su identidad contexto rico y dinámico - a partir del cual uno trata de adaptarse y construirse constantemente.

Si la cultura puede constituir un recurso muy valioso en la construcción 
de nuestra identidad, ésta puede constituirse también en un obstáculo importante, según la utilización que hagamos de ella, la cultura puede convertirse en una negación del individuo. Si las culturas son las puertas abiertas al desarrollo humano, al mismo tiempo, el etnocentrismo puede convertirlas en las murallas, sobre las cuales se apoyan las afirmaciones de identidades extremas que provocan los efectos perversos, que justifican las injusticias de todo tipo en la relación de nosotros con los otros.

Hacemos referencia a la cultura en plural, mas allá de toda clasificación jerárquica. Cada pueblo busca responder a las preguntas que le dan sentido a sus existencias. Todos los pueblos tratan de explicarse la vida, la muerte, la salud, la enfermedad y sus relaciones con la naturaleza.

Igualmente, se preguntan sobre la importancia de cómo transmitir a sus descendientes, sus visiones del mundo, sus conocimientos en diferentes dominios y sus sistemas de valores que les permiten construir una coherencia en sus existencias. Todo este vasto proceso de transmisión a través de la educación, en su sentido más amplio, constituye la cultura que caracteriza a cada pueblo.

La cultura se traduce bajo las formas de las tradiciones y los comportamientos que se expresan a través de los sistemas de símbolos, de códigos, de valores, de técnicas etc. Todo este proceso es fundador de identidades. Es a partir del contexto de referencia cultural de origen, que los individuos pueden «negociar»constantemente las influencias, los aprendizajes y adaptarse a las imposiciones culturales, en relación con su propia historia cotidiana. Es en este proceso, donde se tejen las relaciones entre el individuo y su cultura de origen confrontada a las interacciones con otras culturas, que desbordan ampliamente todo determinismo cultural.

Del Etnocentrismo a la Descentración Cultural: En el caso de la escuela, como lo afirma L.E.López: muchas veces son los maestros diplomados y capacitados, productos del sistema educativo, a quienes se trata de modificar, en la medida que, ellos han estado preparados para ocuparse de estudiantes, cuyo perfil difiere bastante del pequeño campesino o del niño que habla una lengua indígena. (LÓPEZ, 1990: 347).

El cuerpo de profesores puede convertirse en el principal obstáculo a la aplicación y al desarrollo de los programas de educación bilingüe e intercultural. No se puede limitar en dar a los maestros, una formación estrictamente técnica pedagógica; hay que realizar tan bien, un trabajo de concientización y sensibilización, a fin de que puedan adquirir una nueva percepción, tanto de las posibilidades reales de aprendizaje y de desarrollo intelectual de sus alumnos, como también de los recursos potenciales de las culturas indígenas, en el marco de la revalorización de sus lenguas y de sus culturas. Este objetivo impregna la perspectiva intercultural e implica a todos los participantes de los programas. La sensibilización e identificación de los maestros con el proyecto, son condiciones fundamentales. 
A partir de estos antecedentes, imaginar la aplicación de la reflexión intercultural supone, como primera consideración: un trabajo de descentración cultural de los actores. Esto evitará, en gran parte, la reproducción de esquemas mentales de categorización y de jerarquización de valores; es el esfuerzo cotidiano de ponemos en el lugar del «otro», para tratar de comprender los puntos comunes que no asemejan. Alejados por la reflexión intercultural de los prejuicios, podremos construir conjuntamente los saberes necesarios, para resolver nuestras necesidades actuales e imaginar las repuestas a los desafíos futuros.

La descentración cultural, como una respuesta al etnocentrismo que nos impregna, debe constituir un trabajo permanente, en tanto que reflexión y práctica cotidiana, sobre nosotros y sobre el mundo exterior. Esta práctica constituye la unica posibilidad de llevar adelante la aplicación de la interculturalidad, que nos permita construir un real diálogo de culturas.

\section{De La EvangelizaciónA La Alfabetizacion Bilingüe}

¿n estos pueblos, como por todas partes en el mundo, encontramos máquinas de coser Singer. Una companía comercial es capaz, en una sola generación, de vender su mercadería en el mundo entero, en tanto que la iglesia, después de 19 siglos, no ha podido de la misma manera, hacer conocer el evangelio (William Cameron Towsend, Fundador del Instituto lingǘstico de Verano -ILV - In: WALUS, E.\& BENNETHAM.,1972,p. 6).

Una alfabetización en castellano y en portugués fue desarrollada por los Jesuitas para evangelizar a los indígenas, en los teritorios de las Misiones en el Paraguay y el Brasil en el siglo XVIII. En ese contexto histórico, una educación bilingüe y textos de gramática e incluso diccionarios de las lenguas indígenas (Tupí-Guaraní) fueron implementados. Existe en la actualidad, una similitud, guardando las distancias históricas, con las misiones protestantes fundamentalistas norteamericanas del Instituto Lingüístico de Verano (ILV/SIL): con sus programas de alfabetización, limitada a la traducción al castellano o al portugués de textos religiosos y en su objetivo de conversión de los indígenas a una religión y a la cultura occidental, en el cuadro de una política de asimilación de los indígenas, por parte de los Estados dominantes. Más allá de la intención de los misioneros, la alfabetización realizada en el curso de este período, permitió la implantación precoz en el Paraguay, de un sistema de educación bilingüe (castellano-guaraní), sistema que tiene una gran influencia hasta nuestros días.

Hacia el fin del siglo XIX, fue la época de la hegemonía inglesa, en los sectores económicos mas importantes. El protestantismo, es en tanto que ideología y práctica religiosa, el instrumento ideológico, que sirvio como uno de los agentes de la penetración de los intereses hegemónicos norteamericanos. 
En el ámbito ideológico, el Panamericanismo protestante busca imponerse con relación al Panhispanismo católico, herencia colonial adoptada por la gran mayoría de las nuevas repúblicas latino americanas. América para los americanos», frase celebre de Monroe, traducía la quimérica idea de expandir y sobretodo imponer los valores y el modo de vida de la América anglosajona sobre todo el continente. Este proyecto, que tenía que ser precedido por la difusión masiva del protestantismo, no logro cristalizarse como se había previsto inicialmente. (MARIN,1992).

Durante los anos veinte, las iglesias evangelicas, nacionales y misioneras descubrirán un terreno virgen, donde ellas podían lograr rápidamente un gran número de conversiones entre los indígenas, sobre quienes, la expansión de la sociedad industrial producía una gran destrucción de la armonía social de sus mundos. Las congregaciones religiosas ya establecidas, comenzaron a activarse en la época, en la que, los gobiernos populistas nacionalizaban las escuelas primarias y secundarias. Las misiones canalizaron sus programas proselitistas hacia los sectores, donde no llegaba la presencia del Estado. Con el apoyo de los gobiernos, algunas misiones norteamericanas, como «a Pioner Missionary Agency» se instalaron en la Huazteca mexicana en 1930, en 1940 lo hicieron en el Brasil, con «a Misión de la Nuevas Tribus «de la Convención Bautista del Sur de los Estados Unidos y en Venezuela en 1943.En el Perú, en 1927, la "South American Indian Mission» (SAM) estableció su sede en Iquitos, capital de la Amazonía peruana, centrando su trabajo sobre el plano escolar. La más famosa entre ellas, fue el Instituto Lingüístico de Verano (ILV), conocido también como Summer Linguistics Institute (SIL), creada por William Cameron Townsend, un ex misionero, con experiencia sobre los indígenas de Guatemala, inició sus actividades en México en 1937.

Durante «a guerra fría», que se inició al fin de la segunda guerra mundial, el Protestantismo fue portador de un mensaje anticomunista, mensaje que fue todavía mas desarrollado al final de los anos cincuenta, para bloquear y limitar la influencia de la triunfante revolución cubana. Este discurso se transforma en una cruzada ideológica, precediendo y anticipándose al fracaso de la política norteamericana.

Marginalizados por el desarrollo industrial, victimas del parasitismo de las ciudades, los indígenas constituyeron un motivo de preocupación para los gobiernos de los anos treinta. Su potencial social y político y el carácter agrario de la revolución mexicana eran considerados como un precedente, que había que tener en cuenta. Fue precisamente en México, donde se crea el primer Instituto Indigenista, que realiza en 1940, en Patzcuaro, el primer Congreso de Indigenistas de América Latina, presidido por Moisés Sanz, asesor del gobierno mexicano, que abrirá las puertas de México al ILV. Ya en el siglo XIX, las sociedades protestantes europeas consideraban que el sector indígena - animista», constituía un territorio exclusivo de su acción (MARIN, 1992). 
En América Latina, las primeras iniciativas de educación bilingüe, se desarrollaron sobre la base de programas de alfabetización destinados a los pueblos indígenas e n 1937 en México y en los años siguientes en varios paises de América Latina y sobretodo en América del Sur, en los años cincuenta. Estos programas han sido realizados principalmente por los misioneros norteamericanos del Protestantismo Fondamentalista del ILV. Esta institución religiosa ha tenido, en esta época, una cobertura académica, que le ha permitido firmar acuerdos con los gobiernos, como el del Perú en 1945, con el Ministerio de Educación, para desarrollar un programa de alfabetización destinado a los pueblos indígenas de la Amazonía, en 1953 con

Ecuador con el Ministerio de Educación, en 1955, en Bolivia, con el Ministerio de Educación y Cultura. Al principio de los anos sesenta, la sede boliviana cubría también el noroeste argentino y el territorio paraguayo del Chaco.

En el Brasil en 1956, con la Fundación Nacional del Indio (FUNAI) del Ministerio del Interior, en Colombia en 1962, con el Ministerio del Gobierno, en Surinam en 1967, con el Ministerio de Educación. En 1976 se instalarán en Chile y en Argentina. En 1972, el ILV trabajaba con 250 grupos etnolingüísticos en América Latina, lo que equivalía al 47\% de los pueblos indígenas. El número de sus colaboradores creció en diez anos (1975-1985) de 2,150 a 8,000.

Los gobiernos de estos países encontraron en los misioneros los aliados indiscutibles de sus políticas de «ntegración Nacional», destinadas al control politico, económico y cultural de los indígenas. En realidad las llamadas políticas de «ntegración»se redujeron y se reducen en gran parte, hoy en día, a políticas de simple asimilación en el cuadro del modelo politico del EstadoNación, incapaz de reconocer y respetar la diversidad cultural y lingüística que impregna cada pais (MARIN, 1992).

La alfabetización bilingüe propuesta está centrada principalmente sobre la imposición del castellano o del portugués, como lenguas oficiales y a la traducción en lenguas indígenas de los textos del Nuevo Testamento, con el fin de facilitar su evangelización, objetivo fundamental del ILV. La formación de maestros indígenas bilingües se realizo dentro de esta perspectiva de alfabetización y evangelización. Los primeros ejemplos de una formación de este tipo se remontan a los anos cincuenta, época en la que se organizan en México, los primeros cursos para aprender a los indígenas a escribir en sus lenguas, a fin que ellos puedan enseguida traducir y utilizar la Biblia y así servirse de los manuales de lectura redactados por los misioneros lingüistas del ILV (SSTOLL, 1989; MARIN, 1992).Esta estrategia debía ser ulteriormente adoptada en la mayoría de los países de la región, en el Perú, por ejemplo, el primer curso de este tipo se realizo durante el verano de 1953: once jóvenes indígenas provenientes de seis grupos etnolingüísticos de la región amazonica participaron y comenzaron, en el mismo año, a difundir una educación bilingüe a 270 personas de sus comunidades de origen. (LARRON et Al., 1979). 
A partir de los anos 60 los cursos de formación se multiplicaron y diversificaron, en Bolivia por ejemplo, en el cuadro de un proyecto de educación en el medio rural financiado por la Agencia de Ayuda de los Estados Unidos (USAID), proyecto centrado en la formación de maestros que logro formar de 1978 a 1980,muchos maestros de escuelas. Estos cursos estaban caracterizados por dos aspectos esenciales: su corta duración y su carácter funcional, sobre todo limitados a la traducción en lenguas indígenas de ciertos aspectos de la cultura occidental y mensajes de la ideología protestante.

La alfabetización bilingüe en América del Sur, ha sido desarrollada principalmente por misiones religiosas, en el interés de difundir su ideología religiosa, bajo la cobertura de programas escolares. La alfabetización en castellano o portugués que fue adoptada por los Estados que apoyaron la instalación de los grupos misioneros, se realizo a fin de que éstos les ayuden en la asimilación de los pueblos indígenas dentro del marco de sus políticas mal llamadas de integración nacional». La alianza de los Estados y las misiones se consolidó en el eje evangelización y alfabetización.

La escuela oficial y la sociedad multicultural en America Latina, la escuela oficial es tributaria del modelo occidental. Desde el siglo XIX, la escuela se institucionaliza paralelamente a la constitución de las repúblicas. En el siglo XX, Esta educación oficial y los programas de alfabetización prueban de implantar sistemas de valores extranjeros a las culturas indígenas, a pesar de la resistencia cultural y la represión que provocaron, el Occidente impuso su visión del mundo, su lengua y su cultura (MONTOYA,1990, 1998; MARIN, 1992, 1994; UGARTECHE, 1999).

Para el conjunto de pueblos indígenas de las Americas, la cultura está considerada como el producto de las relaciones íntimas que desarrollan el hombre y la naturaleza. La naturaleza es considerada como un sistema viviente, del cual el hombre forma parte (DESCOLA, 1987; GASCHÉ, 1999; GIRALDO, 2000; MONTOYA, 1990; MARIN, 1996; NARBY, 1995). Habría que preguntarse, si al imponer el sistema de educación oficial occidental, no tomamos el riesgo de dar las espaldas a la herencia cultural indígena?

En razón de una larga historia de genocidios y etnocidios sufridos por esos pueblos, la educación oficial se traduce en un proceso de «desindianización» para los pueblos indígenas. (DASEN \& MARIN, 1996: 133135). Desde el principio del siglo XX, emerge en América del Sur, un pensamiento político, en el cual, la escuela es concebida como una intermediaria importante del cambio social. Las influencias del marxismo, de orígen europeo, fueron retomadas y adaptadas a las realidades de esta parte de América. En el Perú, José Carlos Mariategui escribía antes de 1930, su obra «emas de la educación», texto en el cual, él presenta la educación, como el eje del pensamiento económico y político (IBANEZ, 1993; MARIATEGUI, 1970).

En los anos 70, en el Brasil, Paulo Freire publica en 1969, «a educación 
como práctica de la libertad «y en 1970 «edagogía del Oprimido», obras en las cuales, él desarrolla su reflexión sobre la educación popular. Su innovación educativa es concebida en tanto que instrumento de liberación del individuo y como una reflexión sobre los cambios fundamentales en la sociedad (FREIRE, 1969, 1970).

De la educación bilingüe a la perspectiva Intercultural en la educación en América del Sur. Al principio de los años setenta, las federaciones indígenas asumen la defensa y el reconocimiento de sus territorios y al final de esta década, la defensa de las lenguas y las culturas indígenas, que permitirán el surgimiento de una nueva alternativa educativa, que se desarmollará para anteponerse a las políticas asimilacionistas de los Estados.

En los años ochenta, una nueva reflexión en la educación toma forma y se desarrollan en América Latina, los programas de educación bilingüe e intercultural. Estos programas buscan construir una escuela apropiada a los intereses y el respeto por las lenguas y la revalorización de las culturas de los pueblos indígenas de este continente.

En el ámbito oficial, los indígenas son considerados como un obstáculo para el «desarrollo»y el progreso»de los países. Los indígenas son considerados como la expresión de culturas atrasadas, que constituyen una barrera a la modernización. La problemática indígena ha estado construida principalmente sobre una doble situación de opresión de los pueblos indígenas, marcadas por la explotación económica y la discriminación racial, linguistica y cultural (VARESE, 1985, 1987).

La perspectiva intercultural se inscribe como el eje principal de la respuesta a la interrogante, de cómo construir una educación adaptada a los pueblos indígenas de América Latina. Este desafío debe proponerse en función del respeto de la diversidad lingüística y cultural, que deben tener en cuenta los programas educativos desarrollados por los Estados.

Todos estos proyectos son desarrollados por equipos multi-diciplinarios (antropólogos, lingüistas, pedagogos, ingenieros, biólogos etc.) Estos equipos pertenecen a diferentes instituciones y organizaciones que proponen los programas, Instituciones universitarias, organizaciones religiosas, organizaciones no-gubernamentales, muchas veces en asociación con organizaciones indígenas o estatales (DASEN \& MARIN, 1996; GASCHÉ, 1989, 1998, 199= 9; MONTOYA, 1990, 1998).

En el caso particular de la Amazonía peruana, el primer programa de educación bilingüe e intercultural se desarrolló en 1975, como proyecto de revalorización de lenguas y saberes de las culturas indígenas. La idea del respeto de los derechos de estos pueblos y de sus identidades, fue tomada como punto de partida de una política de integración democrática al interior de una sociedad multicultural, característica que impregna a muchos países de América Latina. La participación de organizaciones indígenas a estos progra- 
mas, permite abrir el debate sobre el problema relativo a la posesión de sus territorios, sín los cuales, estas culturas están condenadas a la extinción. Cuál es el lugar de los indígenas en el presente y en el futuro económico, social y cultural de estos paises? Esta importante interrogante queda sin respuesta, esta pregunta nos hace comprender que, la dimensión política de la educación tiene una gran importancia en la historia de estos pueblos.

GASCHÉ afirma que: «La educación en los pueblos indígenas de América Latina, vista desde una perspectiva más general de una educación intercultural y bilingüe, desborda hoy en día, la problemática concerniente a los indígenas. Esta debe interesar a todos los países que albergan poblaciones minoritarias con características sociales, religiosas, lingüísticas y culturales que les diferencian de una sociedad, religión, lengua o cultura que pretenden ser representativos de «a nación», en tanto que, mito unificador patrocinado por el Estado; de este hecho, este se atribuye el derecho de ocupar una posición dominante en el panorama pluricultural del país»(GASCHÉ, 1999:1). En América Latina, las tradiciones amerindias han tenido contactos con otras culturas, principalmente con España y Portugal y las diferentes culturas europeas, Árabes, africanas y asiáticas, en circunstancias históricas diversas.

Este proceso ha permitido tejer múltiples redes culturales y sociales en el pasado, como actualmente. Estas relaciones son el producto de multiples interacciones, tanto pacíficas como conflictivas. Los pueblos indígenas de América, cuentan aproximadamente con 50 millones de personas; estos pueblos determinan la configuración histórica y cultural del continente. En consecuencia tiene un papel a jugar en el futuro de la cultura y de la educación de sus pueblos. Estos últimos anos, los gobiernos latinoamericanos han tomado conciencia de esta realidad; lo que explica que asistamos actual mente, al desarrollo de diferentes iniciativas (LÓPEZ, 1993: 8; GODENZZI, 1996; GASCHÉ, 1998).

Estos pueblos se convierten en sujetos de su propia historia, después de haber sido objetos de la política de asimilación forzada, impuesta por los Estados-Nación.

Constituidos en federaciones y apoyados por las Organizaciones NoGubernamentales (ONGs), exigen el derecho a sus territorios que ocupan después de tiempos ancestrales y aspiran a una educación que revalorice sus lenguas y culturas; educación capaz de responder a sus realidades ecológicas y a sus necesidades cotidianas. Los gobiernos han realizado algunas concesiones y reconocido ciertos derechos. Las reivindicaciones políticas concernientes a la educación son fundamentales, desde el momento que la escuela, en cierta medida, es portadora de un modelo de sociedad determinada. Estos programas no se limitan solamente a los paises donde la población indígena es cuantitativamente importante (México, Guatemala, Ecuador, Perú y Bolivia) sino también a países como Colombia y Chile, con una minoritaria población 
indígena, donde constatamos la creación de programas de educación bilingüe e intercultural o intercultural y bilingüe, tal como son denominadas en los diferentes paises de la región.

En el contexto latinoamericano, la reflexión intercultural, como eje de la educación, no se limita a una simple reflexión teórica; si no ésta constituye la base de investigaciones asociadas a una práctica en relación con realidades concretas, como es el caso de la elaboración de programas de educación, donde el bilingüismo y la interculturalidad son los fundamentos de la práctica educativa (MONTOYA, 1990; GASCHÉ, 1989, 1998, 1999; GODENZZI, 1996). La perspectiva intercultural no busca encerrar a los individuos en sus identidades culturales, bajo el pretexto de liberarlos del dominio occidental y de la standarización cultural, sino, de construir un nuevo paradigma basado sobre los intercambios culturales, dentro de una perspectiva y en condiciones de complementariedad e igualdad, respetando la dignidad de los participantes, a esta experiencia basados en el reconocimiento y a la valorización mutua, en el ambito social, económico, político, religioso y cultural. En el caso de los pueblos indígenas, el respeto de las personas que pertenecen a una cultura determinada es fundamental.

La interculturalidad es concebida como uno de los fundamentos, de un proyecto democrático, al interior de las politicas educativas; esta impregna la sociedad y la democracia en el sentido más amplio. La interculturalidad como proyecto de diálogo de culturas está asociada a los programas educativos, en la busqueda de reforzar las identidades propias a los pueblos indígenas. Desde el principio la relación entre colonos y pueblos colonizados, solo podían provocar conflictos de poder. La condicion de una verdadera relación intercultural, en estas condiciones, no podía ser viable. Han sido necesarios siglos de injusticias y sufrimientos para imaginar el reconocimiento de los indígenas y sus culturas. Esta condición histórica previa, se ha tenido que traducir en el rechazo de los prejuicios, en un cambio de actitudes y mentalidades, como condición esencial para imaginar la interculturalidad en la práctica. La descentración cultural, como una trabajo sobre nosotros mismos, que facilite nuestra relación con los otros, es un trabajo cotidiano que nos liberar de las perversidades del etnocentrismo, que nos impregna a todos. En el plano educativo, esta premisa nos puede ayudar a no imponer contenidos y a definidos, desde nuestro etnocentrismo, en nombre de la interculturalidad (GODENZZI, 1994:172).

Para algunos, el concepto de interculturalidad representa sobre todo la relación entre una cultura indígena y la cultura occidental, pero la multiculturalidad que impregna las sociedades contemporáneas, desborda ampliamente esta visión reduccionista. En América Latina en los años ochenta, se habla de educación bi-cultural. Actualmente la opción se dirige hacia una educación intercultural (JUNG, 1992: 62-65). 
Introducción a la reflexión intercultural en el contexto de América del Sur. Desde el punto de vista teórico, se puede concebir la educación intercultural, como una proposición a partir de un contexto político dado, que busca orientar a los actores educativos a reaccionar a la subordinación impuesta, por los sistemas de conocimientos y los sistemas de valores de la cultura dominante. Según GODENZZI (1996), la interculturalidad, como reflexión de base en los programas educativos, tiene en cuenta, ciertos hechos que podríamos resumirlos de la manera siguiente:

- La finalidad de la reflexión intercultural se plantea en la perspectiva de construir relaciones justas en términos económicos, sociales, políticos y culturales. El paso de una situación de igualdad es el resultado de un proceso de negociación social en el curso del cual los participantes, sobre todo los pueblos indígenas, los mas marginalizados, logran construir su autonomía y logran definir sus derechos en tanto que ciudadanos.

- La interculturalidad busca construir relaciones pedagógicas y un diálogo en términos de igualdad, donde se articulen de manera creativa los diversos conocimientos y los diferentes sistemas de valores.

- El paso de una situación anterior determinada, hacia una situación deseada a través de la aplicación de la reflexión intercultural, estimula la acción educativa hacia una negociación permanente entre los diferentes actores. (GODENZZI, 1996: 14-15).

BRANDÃO (1991:32-45) sugiere que, para abordar el análisis de la interacción de las culturas, cabe distinguir dos enfoques: uno de tipo sociopolítico, centrado sobre el poder y el otro de tipo simbólico, centrado sobre el saber. El primer enfoque considera la cultura, como un conjunto des repuestas dadas por el hombre para satisfacer sus necesidades de reproducción y de organización social, y como un instrumento de poder que, a través de diversas estrategias, legitima un orden social existente, se reproduce y transforma. El segundo enfoque, comprende la cultura como una creación solidaria gobernada por las necesidades del consenso, como saber y comunicación, que permite acuerdos básicos sobre el significado de los códigos y del sentido del mundo.

Estas dos maneras de comprender las realidades culturales están presentes y describen aspectos claves que deben ser considerados en todo estudio sobre las propuestas interculturales (GODENZZI, 1996: 16).

Actualmente, la tendencia dominante de los Estados-Nación en América Latina y en América del Sur, se inscriben en el marco de la globalización bajo la orientación de políticas y de la ideología neo-liberal, que imponen los mitos del progreso y del desarrollo económico y del pensamiento único hegemónico.

Bajo estos antecedentes, asistimos a la negación y a la destrucción arbitraria de tradiciones culturales, hecho que erosiona la dignidad y los derechos fundamentales de las personas y de los pueblos indígenas. Este proceso de 
globalización de la economía no posee un proyecto de sociedad humana, donde las respuestas concretas a los desafíos contemporáneos del medio ambiente y de la dignidad humana, me parecen indispensables.

En el contexto latinoamericano, hay que imaginar la creación de proyectos capaces de responder a las necesidades fundamentales de los sectores populares. En América Latina, la interculturalidad, es todavía en gran parte, una respuesta en construcción, limitada a concebir una educación «destinada a los indígenas». En Europa, actualmente, la reflexión de la interculturalidad, solo se limita a los inmigrantes; sin tener en cuenta que la diversidad sociocultural y lingüística de la multiculturalidad ya impregna a nuestras sociedades, y en consecuencia, es por tanto necesario abandonar el lugar marginal que este enfoque ocupa y asumirlo en la búsqueda de la construcción de una educación adaptada a la sociedades multiculturales en las que hoy vivimos. (ALEMANN-GHIONDA, 1999).

Más allá del continente americano, tenemos el ejemplo de los pueblos autóctonos de la Polinesia, de Hawai, de Nueva Zelandia y de los aborígenes de Australia, que han sufrido grandes mutaciones, perpetradas por el colonialismo y el post-colonialismo. Actualmente, la agresión cultural continúa por las enormes fuerzas de la uniformización cultural, que representan las sociedades industriales dominantes, la mayoría de las naciones insulares del Pacífico sur se encuentran dentro de esta realidad. Aún cuando los pueblos indígenas son numéricamente y políticamente más importantes, sus tradiciones y valores culturales están amenazados (TEASDALE \& LITTLE, 1995, TEASDALE,1999).

Según TEASDALE (1999), algunas soluciones comienzan a emerger de la parte de ciertas culturas autóctonas. Las condiciones socioculturales varían enormemente, de ahí que, las soluciones planteadas en un contexto, no funcionen necesariamente en otro, a pesar de estas consideraciones, las tendencias más importantes que se afirman, puedan ayudarnos a comprender mejor el contexto latinoamericano.

Es indispensable que los pueblos autóctonos posean verdaderamente la libertad de tomar sus propias decisiones concernientes a la educación. Ellos tienen perfectamente el pleno derecho y la autoridad sobre la educación, sino también sobre todas las otras instituciones sociales, económicas, políticas que influencian directamente sobre sus vidas.

La preservación de las lenguas es vital para la supervivencia de las culturas de los pueblos autóctonos. Tendrían que beneficiar de un apoyo para mantener y desarrollar sus lenguas. Los pueblos indígenas tendrían que tener la libertad de volver a pensar la escuela, en función de sus propios parámetros culturales y disponer de los recursos que les aseguren la posibilidad de escoger los modelos de escolarización, que favorezcan su desarrollo lingüístico y cultural. 
Reconociendo los derechos culturales de los pueblos indígenas, los educadores no-indígenas deberían rechazar la suposición de que, el conocimiento occidental sea mas válido o más legítimo que los otros conocimientos, deberían afirmarse igualmente, los saberes tradicionales y «desfolklorizan»los conocimientos indígenas, afirmando que pueden ser parte de la convergencia de saberes, al interior de las escuelas y de las otras instituciones de educación. lo que asocia la educación formal e informal en la practica

escolar. El enfoque del «sentido negociado», puede servir para ensamblar un sincretismo de saberes, dentro de una perspectiva complementaria entre los sistemas de conocimientos indígenas y occidentales (TEASDALE, 1999).

Plantear esta última característica de la aplicación de la reflexión intercultural implica que, los actores deben realizar un trabajo sobre ellos mismos, para lograr una descentración cultural, que permita crear el dialogo intercultural, dentro de una perspectiva de complementariedad y reciprocidad, que sea capaz de integrar los conocimientos de las culturas que participan en el programa, más allá de las falsas oposiciones, entre la oralidad y la escritura y entre la educación tradicional y la educación occidental, reconociendo la racionalidad propia a cada cultura.

El Programa de Formación de Maestros en Educación Bilingüe e intercultural (PFMB) de Zungarococha, cerca de Iquitos, ciudad en la Amazonía peruana, plantea algunos puntos de referencia para llevar adelante su iniciativa: Asociar la educación tradicional a la escuela occidental, no; No, a un sistema único de escuela; No, a una oposición de la educación tradicional a la escuela moderna» (escuela oficial); No, a una ideología del asistido No, a una simple traducción de la cultura occidental en el cuadro de la educación Bilingüe.

Sí, a un aprendizaje del castellano como segunda lengua Sí, a una educación ligada a la ecología Sí, a una educación asociada a la realidad socioeconómica, política y cultural. (AIDESEP / ISPL / PFMB, 1998).

Podemos encontrar un punto común, en las diferentes iniciativas y en su carácter complementario, lejos de toda oposición falsa entre educación tradicional y educación occidental; entre la oralidad y la escritura; entre la lengua oficial y las lenguas maternas. Otra característica importante, es la preocupación de no reproducir la dominación cultural, a traves de la simple traducción de la cultura occidental en nombre de una supuesta educación bilingüe y de no oponer, ni jerarquizar los saberes de los diferentes actores en estas iniciativas: Indudablemente, la descentración cultural, es una preocupación constante, para garantizar la construcción de un diálogo de culturas, como eje de la interculturalidad. 


\section{Conclusiones}

La experiencia de la reflexión intercultural latinoamericana y en Sudamérica está en construcción. No podemos hablar de una experiencia tipo, ya que ésta depende del contexto de cada país y de cada región, y de quienes llevan adelante la experiencia sobre el terreno.

Las actuales iniciativas existentes en los diferentes países están asociadas a políticas sociales y educativas, en tanto referencia en el debate pedagógico, en la formación y la creación de programas y de material didáctico. El eje «educación y democracia», constituye un debate obligado. Esta reflexión es considerada como el fundamento de una escuela adaptada a la diversidad cultural y lingüística de cada país, una escuela capaz de poner en valor y de coordinar las diferentes culturas existentes.

La interculturalidad no es la simple transmisión de programas, ya que esta perspectiva interdisciplinaria permite la articulación de diferentes aportes a los nuevos conocimientos, en favor de una construcción global, elaborada por el conjunto de los actores alrededor de un mismo sujeto. De hecho, la interculturalidad enriquece los programas del conjunto con los aportes de cada cultura, a partir de la interculturalidad vivida en la práctica (HOWARD-MALVERDE, 1996).

Aplicada en el dominio de la educación, una aproximación de la realidad observable por las diferentes culturas, implica el estudio de las condiciones de la transmisión de saberes en diferentes tipos de aprendizajes. El estudio transversal de la educación, en el sentido más amplio, permite comprender mejor y poner en valor la educación tradicional, formal e informal en su complementaridad.

Cómo imaginar la colaboración de profesores formados en la universidad occidental, compartiendo con los especialistas indígenas en su lengua y su cultura, en vista de elaborar un programa educativo intercultural? Cómo evitar el riesgo de una imposición del contenido, que se limite a la cultura oficial en los programas, que pretenden presentarse como interculturales? Cómo se podría lograr la necesaria descentración cultural de los actores? Cómo coordinar las experiencias vividas, en la perspectiva de compartir saberes, como base en el diálogo de culturas? Estas son preguntas a responder y una realidad por construir.

Es posible de imaginar el papel importante que puede jugar las emisiones de radio, difundida en lenguas indígenas, como agente de socialización familiar e imaginar que puedan difundirse estos programas en el ámbito internacional. Las experiencias de Bolivia y Ecuador, son una buena demostración de la puesta en practica de la interculturalidad, tanto en el ámbito lingüístico como cultural (ALBO \& EMILIO, 1990; ALBO, 1995; 
AMADIO, 1989, 1990; AMADIO, VARESSE \& PICON, 1987; CHUQUIMANTARI \& QUISPE, 1996; HOWARD-MALVERDE, 1996; MOYA, 1990; VARESE, 1987, 1990).

La descentración cultural, constituye un paso obligado para los actores, que participan en la aplicación de la interculturalidad, en las diferentes iniciativas existentes. El rechazo de los prejuicios que nos permita un cambio de actitud, es un acto necesario, que evite la reproducción de esquemas mentales de jerarquización de valores; este acto corresponde a un esfuerzo fundamental, constante y a un trabajo sobre nosotros mismos, que nos facilite el encuentro y un diálogo justo y respetuoso con los otros.

La descentración cultural, debe permitirnos de imaginarnos, en el lugar y en la situación de los otros, para así tratar de comprender los puntos comunes que nos unen, en tanto miembros de una sola especie humana. El papel de la socialización en la escuela podría constituir una estimulación muy eficaz en la descentralización cultural.

Crear las condiciones que nos permitan vivir juntos, con nuestras diferencias, debería constituir un desafío colectivo. En efecto, a partir de una reflexión intercultural de los prejuicios, puede ser posible construir conjuntamente, los saberes necesarios para satisfacer nuestras necesidades actuales e imaginar las respuestas a los desafíos futuros. Los pueblos indígenas forman parte de las sociedades multiculturales de las diferentes regiones de América Latina y encontraran necesariamente en la riqueza del intercambio de sus diversidades con las sociedades llamadas nacionales», los materiales necesarios para una construcción social y democrática, respetuosa de la dignidad humana.

Las interrogantes planteadas por el problema de los pueblos indígenas del continente americano, pueden compararse, guardando las distancias y teniendo en cuenta su especificidad, con los problemas que encuentran el respeto de la diversidad lingüística y cultural en otros continentes, como hemos visto en el caso de Oceania, o el de Europa, confrontadas al desafío de la integración de sus minorías étnicas o nacionales y a los inmigrantes.

La democracia actual, sumergida en el proceso de la globalización de la economía, encuentra en el desafío de una justa gestión de su pluralidad cultural, lingüística y en la solución de los grandes conflictos socioeconómicos, uno de sus grandes desafíos.

La interculturalidad, es una reflexión necesaria para imaginar, no solamente una educación adaptada y respetuosa de la diversidad de los pueblos indígenas o de los inmigrantes; sino también, una reflexión obligada para la gestión democrática de la sociedad multicultural contemporánea. 


\section{Referencias Bibiliograficas}

ALBO, X.Bolivia plurilingüe: guia para planificadores y

educadores. 2 Vol. La Paz : UNICEF - Centro de Investigación y Promoción de las Culturas Autoctonas, 1995.

ALBO , X. \& EMLLO, L Education etlangues autochtones en Bolivie. Perspectives, 75 (3) 358- 36, 1990

ALLEMANN-GHIONDA, C. Assumer la diversité; socioculturelle et linguistique comme finalité de l'éducation. In C. Alleman-Ghionda (Ed.), Education et diversité socioculturelle (pp.11-27). Paris :Harmattan,1999.

AIDESEP / ISP LORETTO / PFMB. Programa curricular diversificado de educación primaria intercultural bilingüe para los pueblos amazónicos. Iquitos (Pérou): AIDESEP-ISP, 1998.

AMADIO, M.Deux décenies d'éducation bilingue en Amérique Latine (19701990). Perspectives, 1990.

AMADIO, M.La cultura como recurso político : dinámicas y tendencias en América Latina. In: L E.López \& R. Moya (Ed.). Pueblos Indios, Estados y Educación (pp. 27-37). Lima : PEB96 Puno, Proyecto EB I/ MEC-GTZ, Programa Educación Rural Andina (ERA), 1989.

AMADIO, M. , VARESE, S. \& PICON C. Educación y pueblos indígenas en Centro América. Un balance crítico. Santiago de Chile : UNESCO, Oficina Regional de Educación para Aménica Latina y el Caribe (OREALC), 1987.

AMADIO, M. \& LÓPEZ, L Educación bilingüe intercultural en América Latina: guía bibliográfica. La Paz : UNICEF-Centro de Investigación y Promoción de las Culturas Autóctonas, 1993.

AMADIIO, E. Formas de la alteridad. Construcción y difusión de la imágen del indio americano en Europa durante el primer siglo de la conquista de América. Quito : Ediciones Abya, 1993.

ARRATIA, M.-I. Una experiencia piloto en educación intercultural en la región aymara del norte de Chile. Pueblos indígenas y educación. 29-30, 193-212. Quito : Proyecto EBI y Ediciones Abya, 1994.

BRANDÃO, C. A arca de Noe. ApontamenTos sobre sentidos e diferenças a respeito da ideia de cultura. In: P. Suess (Ed.). Culturas e evangelição. São Paulo: Ediçoes Loyola, 1993.

CAMILERI, C. Le relativisme : du culturel à l'interculturel.Paris: Harmattan, 1996. 
TANON \& G. VERMES (Ed.) L'individu et ses cultures. Paris : Harmattan, 1993. CAMPA MENDOZA, V. Las insurrecciones de los pueblos indios en México. La rebelión Zapatista en Chiapas. Durango (México) : Ediciones Cuellar, 1999.

CHIOLDI, F. (Ed.). La educación indígena en América Latina. Mexico/ Guatemala/Ecuador/Perú/Bolivia. Quito/Santiago de Chile : Proyecto EBI (MECGTZ) \& ABYA YALA / UNESCO / Oficina Regional de Educación para América Latina y el Caribe (OREALC), 1990.

CHOMSKI , N. \& DIEWTERICH, H.La Aldea global. Tafalla (Espagne) : Txalaparta, 1998.

CHUQUIMANTARI, N. \& QUISHPE, N. Interculturalidad en las aulas y en los libros. In: J. Godenzzi (Ed). Educacion e interculturalidad en los Andes y la Amazonía. Cusco (Pérou) : Centro de Estudios Regionales Andinos Bartolomé de las Casas, 1996.

CLANET, C. L'Interculturel : introduction aux approches interculturelles en éducation et en sciences humaines. Toulouse : Presses Universitaires du Mirail, 1993.

COCCHI, F. Recherche sur le terrain concernant l'éducation bilingue et interculturelle en Amérique du Sud. Manuscrito no publicado, 2000.

CUCHE, D. Nouveaux regards sur la culture. Evolution d'ne notion en

anthropologie. Sciences Humaines, 1997CUCHE, D. La notion de culture dans les sciences sociales.Paris : La Découverte, 1996.

DASEN, P. \& MARIN, J. La modernité éducative etla conquète du monde. In: D. Hameline et J. Vonche (Ed.). Jean Piaget. Agir et construire. Aux origines de la connaissance chez enfant et le savant. Genève : Faculté de Psychologie et de sciences de l'éducation, Université de Genève / Musée d'ethnographie, 1996.

DEMORGON, J. L'histoire interculturelle des sociétés. Paris : Anthropos, 1998.

DEMROGON, J. \& LIPIANSKI, E.-M.(Ed.). Le guide de l'interculturel en formation. Paris : Retz, 1999.

DEMORGON, J. L'interculturation du monde. Paris: Anthropos, 2000.

DERENS, J.-A. Les Tziganes, indésirables au Kosovo. Le Monde Diplomatique 548, novembre, 8-9, 1999.

DESCOLA, Ph. La nature domestique : Symbolisme et praxis dans l'écologie des Achuar. Paris: Fondation Singer-Polignac / Editions de la Maison des Sciences 
de l'Homme, 1986.

DÍAZ POLANCO, H. La rebelión Zapatista y la Autonomía.México : Editorial Siglo Veinte, 1997.

FERRER, F. L'Espagne des communautés autonomes et les droits linguistiques. In S. Perez (Ed.) La mosaíque linguistique. Regards éducatifs sur les pays industrialisés. Paris : L'Harmattan, 1998.

FORRESTER, V. L'horreur économique. Paris : Fayard, 1996.

FREIRE, P. La educación como practica de la libertad.Montevideo: Ed. Tierra Nueva, 1969.

FREIRE, P. Pedagogía del oprimido. Montevideo : Ed. Tierra Nueva, 1979.

GALE, P. «Donner leur chance à tous ?«Les droits autochtones et l'enseignement du troisième degré en Australie. Perspectives, 1995

GASCHÉ, J. Un concept syntactique de la «culture»et ses avantages : créativité et flexibilité. Manuscrit soumis pour publication, 1999.

GASCHÉ, J. Rapports interculturels entre les peuples indiens et la société nationale : portée politique et pédagogique des varietés de discours. [Texte téléchargé] Accès : http://www.uquebec.ca/diverscité.

GASCHÉ, J. RevalorisatIon culturelle et structure du programme de formation d'instituteurs interculturels et bilingues de la confédération indienne amazonienne AIDESEP et de l'Institut Supérieur Pédagogique LORETO. [Texte téléchargé] Accès : http://www.uquebec.ca / diverscité, 1998b.

GASCHÉ, J. A propos d'une nouvelle expérience d'éducation bilingue au Pérou. Bulletin de la Société Suisse des Américanistes, 53-54, 131-142, 1989.

GIRALDO, J. Gliwa un popolo che non vuole essere venduto. Roma : Fundazione Lelio Basso, 2000.

GLEICH, U. Caracterización de la educación primaria bilingüe intercultural para ninos de habla vernácula. Eschborn (RFA) : Coopération Technique Allemande (GTZ), 1989.

GODENZZI, J. (Ed.). Educación e interculturalidad en los Andes y la Amazonía. Cusco : Centro de Estudios Regionales Andinos Bartolomé de las Casas, 1996. HALAK, J. Education et globalisation. Paris : UNESCO, 1998.

HESS, R. (1998) Une grande aventure de notre temps.In J. Demorgon. L'histoire interculturelle des sociétés (pp. V-XIII). Paris : Anthropos,1998. 
HOBSBAWN, E. L'âge des extrêmes. Histoire du court XXème siècle. Paris : Editions Complexe / Le monde Diplomatique, 1999.

HOWARD-MALVERDE, R. (1996). La interculturalidad vivida : testimonios de mujeres desde el norte de Potosí. In J.Godenzzi Alegre (Ed.). Educación e interculturalidad en los Andes y la Amazonía (pp.115-138). Cusco (Pérou): Centro de Estudios Regionales Andinos Bartolomé de las Casas,1996.

HUNTINGTON, S. Le choc des civilisations. Paris: Editions Odile Jacob, 1997. IBANEZ, A. Para repensar las utopIas. Lima: SurTarea, 1993.Jäggi, C.Les gens du voyage en Suis= se.Lucerne : Editions CaritaS, 1988.

JANODET, L. \& FERREIRA, C. Les Tziganes et les gens du voyage dans la cité. Paris : L'Harmattan, 1992.

JUNG, I. Conflicto cultural y educación. El proyecto de educación bilingüe Puno. Quito: Proyecto Educación Bilingüe (EBI) y Ediciones Abya Yala, 1992.

LANGANEY, A., VAN BIJENBURGH, N. \& SÁNCHEZ-MAZAS, A. Tous parents, tous différents. Paris: Chabaud, 1992.

LAPLANTINE, F. \& NOUSS, A. Le metissage. Paris : Dominos / Flammarion, 1997.

LATOUCHE, S. L'occidentalisation du monde. Paris: Agalma - La Découverte, 1989.

LEWIN, K. Le cas de Yi dans la province de Szeu Chuan (Chine). Perspectives, 96, (4), (687-704), 1995.

LEMPEN, B. La mondialisation sauvage. De la fin du communisme; la tragédie du Kosovo. Lausanne: Favre, 1999.

LIEGEOIS, J.-P. (1994). Roma, Tziganes, voyageurs. Strasbourg : Les Editions du Conseil de l'Europe, 1994.

LPKA, J. \& STAIRS, A. (Ed.). Negociating the culture of indigenous schools. [numéro spécial]. Peabody journal of education.69 (2), 1994.

LONGO, T. Philosophie et politiques Néo-Libérales de l'éducation dans le Chili du Pinochet, 1973-1983. Paris : L'Harmattan, 2001.

LÓPEZ, LLe développement des ressources humaines par et pour l'éducation bilingue interculturelle en Amérique Latine. Perspectives, 75 (3) (345-355)M 1990.

LÓPEZ, L E. \& MOYA, R. Pueblos Indios, estados y educación. Lima : Proyecto 
de educación bilingüe (PEB) - Puno, Proyecto Educación Bilingue interculural (EBI) / MEC- Coopération technique allemande ( $\mathrm{G}=\mathrm{TZ})$, Programa Educación rural andina (ERA), 1989.

MARIATEGUI, J.-C. Temas de educación.Lima : Empresa editora Amauta, 1970.

MARIN, J. Le développement durable et la dimension interculturelle. In: A. Giordan \&J. Denis-Lempereur (Ed.). 12 questions d'actualité sur l'environnement (pp. 127-130). Nice : Ministère de l’Environnement, 1996.

MARIN, J. Dimension historique de l'ethnocentrisme européen dans le processus de la domination coloniale et post-coloniale de l'Amérique. In J. Blomart \& B. Krewer (Ed.), Perspectives de l'interculturel (pp.123=96134). Paris: L'Harmattan, 1994a.

MARIN, J. Ethnocentrisme et racisme dans l'histoire européenne dans le cadre de la conquête de l'Amérique et perspective actuelle. In: C. Allemann Ghionda (Ed. ). Multicultur und Bildung in Europa. Multiculture et éducation en Europe (pp. 181-196). Bern : Peter Lang, 1994b.

MARIN, J. Peuples indigènes, missions religieuses et colonialisme interne dans l'Amazonie péruvienne. Uppsala : Missionsfornkning, Uppsala University, 1992.

MAYER, E. Los alcances de una política de educación bicultural y bilingüe. In M. Amadio , S. Varese \& C. Picon (Ed.). Educación y pueblos indIgenas en Centro América. Un balance crítico . (pp. 27-=37). Santiago de Chile : UNESCO, Oficina Regional para la Educacion de

América Latina y el Caribe (OREALC), 1987.

MC LUHAN, T. Pieds nus sur la terre sacrée. Paris : Denoël, 1971.

MIRANDA VEGA, G. Formación de profesores indígenas para una educación intercultural bilingüe en el norte de Chile,1996.

MONTOYA, R. Multiculturalidad y PolItica. Derechos indígenas, Ciudadanos y Humanos. Lima :Sur Casa de Estudios del Socialismo, 1998.

MONTOYA, R. Por una educación bilingüe en el Perú. Reflexiones sobre cultura y socialismo. Lima : Mosca Azul editores, 1990.

MOYA, R.Dix années d'éducation bilingue et de participation indigène en Equateur. Perspectives, 75 (3) (367-379), 1990.

OMAN, Ch. Los desafíos políticos : globalización y regionalización. Lima;: Fundación Friedrich Ebert, 1996. 
PEREZ, S. (Ed). La mosaìque linguistique. Regards éducatifs sur les pays industrialisés. Paris : L'Harmattan, 1998.

PERREGAUX, C. Odyssea. Accueils et approches interculturelles. Neuchâtel : Commission romande pour les moyens , 1994

PICDARD, E. La question Kurde. PariS: Editions Complexe, 1991.

PRIDMORE, P. L'apprentissage et la scolarité des enfants basarwa (bochimans) au Botswana. Perspectives, 96 (4)( 783-800), 1995.

QUILAQUEO, R. D.(Ed). Educación Intercultural Bilingüe. Actas del Segundo Seminario Latinoamericano. Temuco: Universidad Católica de Temuco, Chile, 1999.

RAMONET, I. Géopolitique du chaoS. Paris : Editions Galilée, 1997.

ROSTKOWSKI, J. Le renouveau Indien aux Etats-Unis. Paris : L'Harmattan, 1973.

TEASDALE, G. Education et culture : quelques mots d'introduction. Perspectives, 96 (4) (645-651), 1995.

TEASDALE, G. \& Little, A. (Ed.). Education et culture. [numéro spéciale] Perspectives, 96= (4) (645-872), 1995.

TEASDALE, G. L'éducation et la survie des petites cultures autochtones. Développement, culture et éducation. Annuaire international de l=92éducation, XLIV, (215-245), 1994.

THODÉ-STUDER, S. Les Tziganes suisses.Lausanne : Editions Réalités Sociales, 1987.

VARESE, S. Défis et perspectives de l'éducation indigène au Mexique. Perspectives, 75 (3) (381- 392), 1990.

VARESE, S. Cultural development in ethnic groups : Anthropological explorations in education. International Social Science Journal, XXXVII, (2) (201-216), 1985.

WARNIER, J.-P. La mondialisation de la culture. Paris: La Découverte. Coll.Repères, 1999.

ZAVALA, J. M. Les indiens Mapuche du Chili. Paris: L'Harmattan, 2000.

ZIMMERMANN, K. Formas de agresión y defensa en el conflicto de las lenguas espanola y portuguesa con las lenguas amerindias. In M. Mörner \& M. ROSDNDHAL (Ed.). Pueblos y medios ambientes amenazados en las Américas, 1995. Stockhölm: Actas del 48 Congreso Internacional de los Americanistas. 
Instituto de Estudios Latinoamericanos, 2000.

ZURIGA, M. , ANSIÒN, J. \& CUEVA, L (Ed.) Educación en poblaciones indígenas: Políticas y estratégias en América Latina. Santiago de Chile :

UNESCO/Instituto Indigenísta Interamericano, 1987.

Recebido em: $13 / 03 / 02$

Aprovado em: 28/05/02 